\title{
AN EFFICIENT FUSION BASED UP-SAMPLING TECHNIQUE FOR RESTORATION OF SPATIALLY COMPRESSED IMAGES
}

\author{
Girinandini Sahoo ${ }^{1}$ and Aditya Acharya ${ }^{2}$ \\ Department of Electronics and Telecommunication Engineering \\ Silicon Institute of Technology, Bhubaneswar, Odisha, India
}

\begin{abstract}
The various up-sampling techniques available in the literature produce blurring artifacts in the upsampled, high resolution images. In order to overcome this problem effectively, an image fusion based interpolation technique is proposed here to restore the high frequency information. The Discrete Cosine Transform interpolation technique preserves low frequency information whereas Discrete Sine Transform preserves high frequency information. Therefore, by fusing the DCT and DST based up-sampled images, more high frequency, relevant information of both the up-sampled images can be preserved in the restored, fused image. The restoration of high frequency information lessens the degree of blurring in the fused image and hence improves its objective and subjective quality. Experimental result shows the proposed method achieves a Peak Signal to Noise Ratio (PSNR) improvement up to 0.9947dB than DCT interpolation and $2.8186 \mathrm{~dB}$ than bicubic interpolation at 4:1 compression ratio.
\end{abstract}

\section{KEYWORDS}

Interpolation, DCT, DST, Image Up-Sampling, Image fusion.

\section{INTRODUCTION}

Resolution of an image has been always an important issue in many images and video processing application, such as video resolution enhancement, feature extraction and image resolution enhancement. Interpolation in image processing is a method to increase the number of pixels in a digital image. Many image interpolation schemes have been proposed in [1]-[4]. There are four well-known interpolation techniques, namely, nearest neighbour, bilinear, bicubic, Lanczos. Discrete Cosine Transform is also playing a significant role in many image processing applications. The conventional discrete cosine transform (DCT) is used to perform twodimensional (2D) interpolation of real sequences. Similarly Discrete Sine Transform (DST) is also used for interpolation. The 2-D Discrete Sine Transform of an image is performed by applying the 1-D Discrete Sine Transform (DST) along the rows of the image first, and again in the columns of the image. Literatures [5]-[7] is schemed a fusion technique. It can earn much better objective quality comparing with simple bilinear, bicubic interpolation in spatial domain and DCT, DST in frequency domain. The DCT interpolation technique preserves low frequency information and DST preserves high frequency information. Therefore by unite the DST and DCT based up-sampled images, both high and low frequency can be preserved in the restored up- 
International Journal on Information Theory (IJIT), Vol.4, No.1, January 2015

sampled image. As a result the fused image contains all the significant information of the input images. The quality of the fused image is superior to any of the input images.

The rest of this paper is organized as follows: Section II provides a brief description of related work which is used for image interpolation. A detail of the proposed fusion technique is described in Section III. Section IV presents the experimental results to show the performance of the proposed method. Section V concludes this paper.

\section{RELATED WORK}

Interpolation filters are used to interpolate new sample values at arbitrary time instants between the existing discrete time samples. Bilinear and Bicubic interpolations are two simple upsampling techniques with low complexity. Bilinear interpolation is the combination of two linear interpolations. The bicubic interpolation gives smother surface than bilinear interpolation. Hence, such interpolation techniques often lead to several types of visual degradation in which the blurring effect is one of the most noticeable artifacts.

DCT based interpolation gives better image's visual quality. [8] It shows a signal or image can be transformed from spatial domain to frequency domain using DCT. The DCT has the property that, for a typical image, most of the visually significant information about the image is concentrated in just a few coefficients of the DCT. For this reason, the DCT is often used in image compression application. It preserves the low frequency components. Again the discrete sine transform is a Fourier related transform similar to discrete Fourier transform (DFT), but using a purely real matrix. It preserves the high frequency components. According to research reported in [9]-[10], interpolation in compressed domain can produce better objective quality such as PSNR than those with a simple bilinear, bicubic, DST based interpolation. Due to zero padding in high frequency components usually lead to blocking artifacts.

Based on above investigation, we develop the weighted fusion technique. It estimates the high frequency components from DST-based interpolation and combines them with low frequency components derived directly from DCT-based interpolation. Thus, a complete frequency domain reconstruction can be achieved by the fusion technique. The key idea in the proposed fusion technique is to restore the high frequency components as well as the low frequency components of the up-sampled image from frequency domain interpolation. Here a better execution is supposed to achieve both in visual and objective quality so as to keep all the important information (low, high frequency) of the interpolated image.

\section{FUSION BASED INTERPOLTION}

As discussed in section 2, any approach in either spatial or frequency domain, is applied to achieve an interpolation that is high in both visual and objective quality. Since the high frequency components are mostly contributed from sharp regions like edges. Therefore, fusion technique is expected to achieve quality improvement in both visual and objective measures. We can also apply fusion technique to both DCT and DST up-sampled images.

\subsection{A. Image up-Sampling in DCT Domain}

To implement up-sampling in DCT domain, we only need to add zeros in the high frequency regions.DCT is calculated for an image of size $M \times N$ is given below: 
$c(u, v)=w(u) w(v) \sum_{x=0}^{M-1} \sum_{y=0}^{N-1} \cos \left(\frac{\pi(2 x+1) u}{2 M}\right) \cos \left(\frac{\pi(2 y+1) v}{2 N}\right)$

where

$$
w(u), w(v)=\left\{\begin{array}{lll}
1 / \sqrt{N} & \text { for } & u=0, v=o \\
2 / \sqrt{N} & \text { for } & u \neq 0, v \neq o
\end{array}\right.
$$

\subsection{B. Image up-Sampling in DST Domain}

The 2-D Discrete Sine Transform of an image is performed by applying the 1-D Discrete Sine Transform (DST) along the rows of the image first, and again in the columns of the image. DST is calculated for an image of size $M \times N$ is give below:

$$
c(u, v)=\sqrt{\frac{2}{N+1}} \sum_{x=0}^{M-1} \sum_{y=0}^{N-1} f(x, y) \cdot \sin \left(\frac{\pi u x}{M+1}\right) \cdot \sin \left(\frac{\pi v y}{N+1}\right)
$$

\subsection{Fusion Based Interpolation Method}

The process of image fusion the good information from each of the given images is fused together to form a resultant image whose quality is superior to any of the input image. Image fusion is applied in every field where image are ought to be analyzed. For example, medical image analysis, microscopic imaging, analysis of image from satellite, remote sensing application, computer vision, robotics etc. Due to limited focus depth of the optical lens it is often not possible to get an image which contains all the relevant objects in focus. To obtain an image with every object in focus a fusion process is required to fuse the images giving a better view for human or machine perception. Block diagram for fusion process is shown in Fig.1.

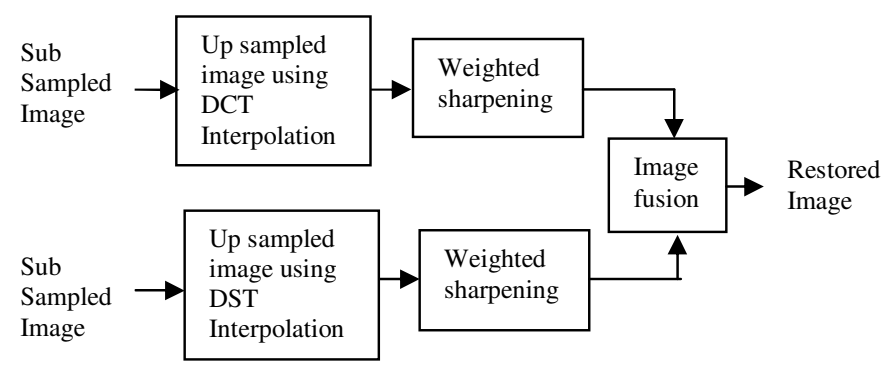

Fig.1: Fusion based Interpolation

The 4:1 compressed image is interpolated by using the DST and DCT based interpolation techniques. Then both DST and DCT up-sampled images are sharpened using Laplacian,

$$
g(x, y)=f(x, y)+k \cdot \nabla^{2}\{f(x, y)\}
$$

where and denote sharpened image and weight factor respectively. The amount of image sharpening is a function of weight factor $\mathrm{k}$ which ranges from 0 to 1 . So, the blurring effect is 
reduced considerably. In this proposed method a better PSNR value is achieved for DCT upsampled image at $\mathrm{k}=0.2$, similarly at $\mathrm{k}=0.01$ for DST up-sampled image the PSNR value remains high. The sharpened images are fused together by using the weighted fusion technique. Finally, the output is obtained in the form of restored image.

$$
\hat{f}(x, y)=k_{1} \cdot g_{1}(x, y)+k_{2} \cdot g_{2}(x, y)
$$

where, and are the sharpened images of DCT and DST up-sampled images respectively. The k1 and $\mathrm{k} 2$ are standardized as $\mathrm{k} 1=0.95, \mathrm{k} 2=0.05$ which is implemented to different images where a high PSNR value is achieved. The flowchart of the proposed method is shown below:

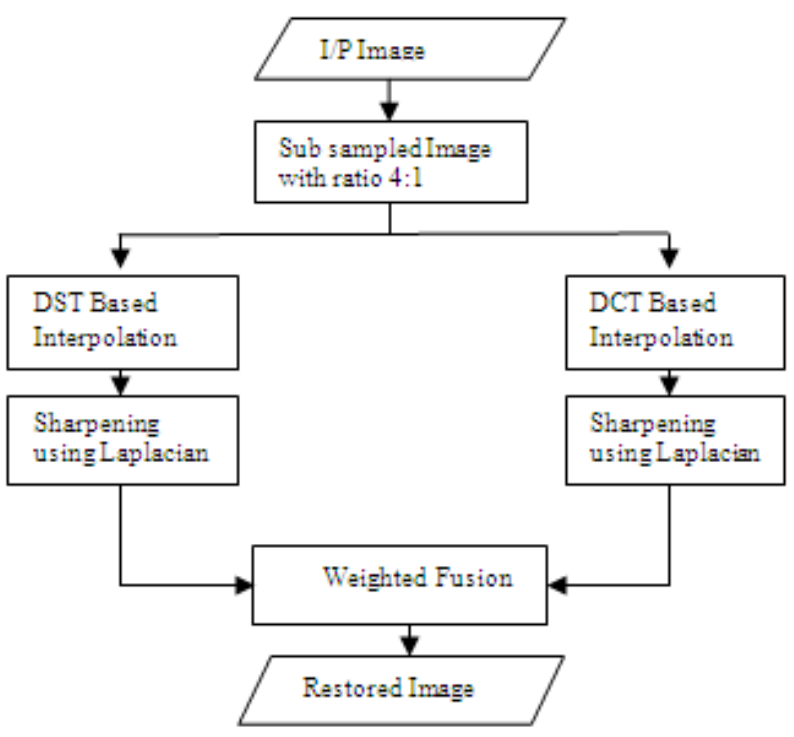

Fig. 2 flowchart of proposed method

\section{RESULTS AND DISCUSSION}

The proposed technique has been tested on several different images and videos. Here, two quality metrics are used to test the performance of the proposed algorithm. One metric is Peak Signal to Noise Ratio (PSNR) and another is Root Mean Square Error (RMSE).

$$
\begin{aligned}
& M S E=\frac{1}{M N} \sum_{x=0}^{M-1 N-1} \sum_{y=0}[\text { Fig. } 2 \text { flowchart of proposed method } \\
& R M S E=\sqrt{M S E} \\
& P S N R_{d B}=10 \log _{10} \frac{(255)^{2}}{M S E}
\end{aligned}
$$


International Journal on Information Theory (IJIT), Vol.4, No.1, January 2015

4.1. Experimental results are tabulated in Table-1, Table-2 and Table-3.

TABLE-1: Describe RMSE values of different interpolation techniques.

\begin{tabular}{|l|l|l|l|l|}
\hline \multirow{2}{*}{ IMAGES } & \multicolumn{4}{|c|}{ RMSE } \\
\cline { 2 - 5 } & Bicubic & DCT & DST & $\begin{array}{l}\text { PROPOSED } \\
\text { METHOD }\end{array}$ \\
\hline Cameraman & 0.0428 & 0.0462 & 0.0543 & 0.0452 \\
\hline Mandrill & 0.0342 & 0.0277 & 0.0374 & 0.0330 \\
\hline Pirate & 0.0282 & 0.0264 & 0.0335 & 0.0258 \\
\hline Lena & 0.0355 & 0.0337 & 0.0435 & 0.0330 \\
\hline Womandarkhair & 0.0086 & 0.0079 & 0.0242 & 0.0077 \\
\hline Barbara & 0.0540 & 0.0551 & 0.0594 & 0.0552 \\
\hline Boat & 0.0318 & 0.0300 & 0.0364 & 0.0293 \\
\hline House & 0.0229 & 0.0221 & 0.0381 & 0.0219 \\
\hline
\end{tabular}

TABLE-2: Comparison between different interpolation techniques

\begin{tabular}{|l|l|l|l|l|}
\hline \multirow{2}{*}{ IMAGES } & \multicolumn{4}{|c|}{ PSNR(dB) } \\
\cline { 2 - 5 } & Bicubic & DCT & DST & $\begin{array}{l}\text { PROPOSED } \\
\text { METHOD }\end{array}$ \\
\hline Cameraman & 26.3320 & 26.7114 & 25.2983 & 26.8954 \\
\hline Mandrill & 29.3243 & 31.1482 & 28.5383 & 32.1429 \\
\hline Pirate & 31.0088 & 31.5531 & 29.4954 & 31.7791 \\
\hline Lena & 28.9962 & 29.4411 & 27.2239 & 29.6291 \\
\hline Womandarkhair & 41.2920 & 42.0834 & 32.3350 & 42.2730 \\
\hline Barbara & 25.3521 & 25.1834 & 24.5267 & 25.1911 \\
\hline Boat & 29.9515 & 30.4661 & 28.7703 & 30.6771 \\
\hline House & 32.7884 & 33.1034 & 28.3715 & 33.1850 \\
\hline
\end{tabular}

TABLE-3: Describe average PSNR values of different interpolation techniques for video intra frame.

\begin{tabular}{|l|l|l|l|l|}
\hline \multirow{2}{*}{ VIDEOS } & \multicolumn{4}{|c|}{ AVERAGE PSNR(dB) } \\
\cline { 2 - 5 } & Bicubic & Lanczos & DCT & $\begin{array}{l}\text { PROPOSED } \\
\text { METHOD }\end{array}$ \\
\hline Coastguard & 26.9185 & 27.2261 & 27.3210 & 27.5092 \\
\hline Football & 25.1483 & 25.3806 & 25.5689 & 25.7384 \\
\hline Bus & 25.2624 & 25.7167 & 25.7945 & 26.0532 \\
\hline Flower & 19.3140 & 19.5015 & 19.4884 & 19.5880 \\
\hline Foreman & 31.6336 & 32.0842 & 32.4367 & 32.7824 \\
\hline Hallmanitor & 25.2644 & 25.6082 & 25.9646 & 26.2007 \\
\hline Ice & 27.6814 & 28.0695 & 28.8634 & 29.2924 \\
\hline News & 25.0578 & 25.4683 & 25.6087 & 25.8619 \\
\hline Salesman & 28.9969 & 29.2500 & 29.3382 & 29.4858 \\
\hline
\end{tabular}


International Journal on Information Theory (IJIT), Vol.4, No.1, January 2015

\subsection{Simulation results}

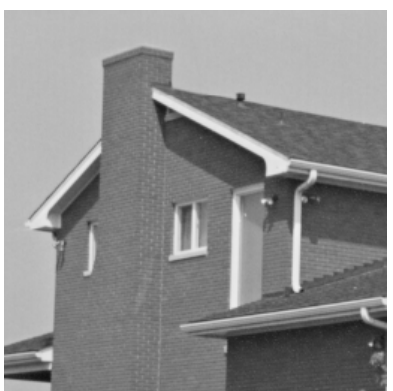

(a)

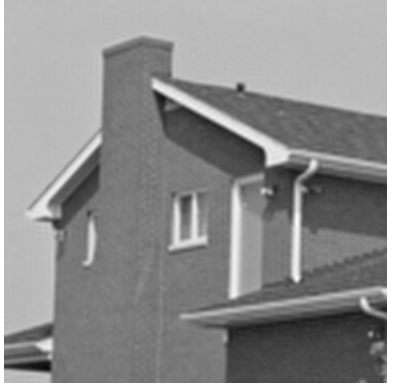

(c)

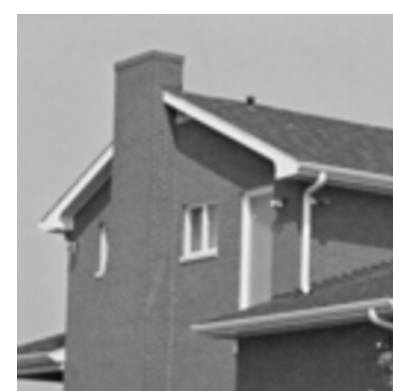

(b)

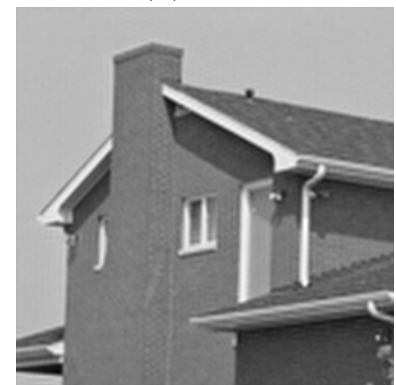

(d)

Fig.3: Subjective Evaluation of House using different Interpolation Techniques: (a) source image (b) Bicubic method (c) DCT method (d) Proposed Method.

From the experimental results, it is observed that the proposed method is giving better result (high PSNR value).

Experiments are also performed with Cameraman and Lena images. Results are shown in Fig.4and Fig. 5 respectively 
International Journal on Information Theory (IJIT), Vol.4, No.1, January 2015

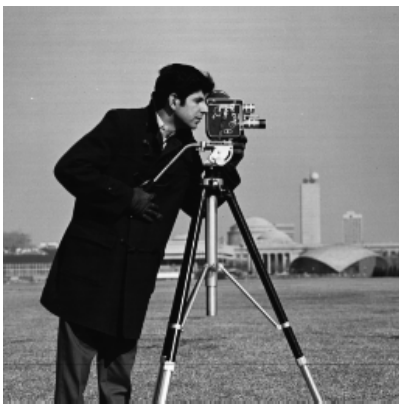

(a)

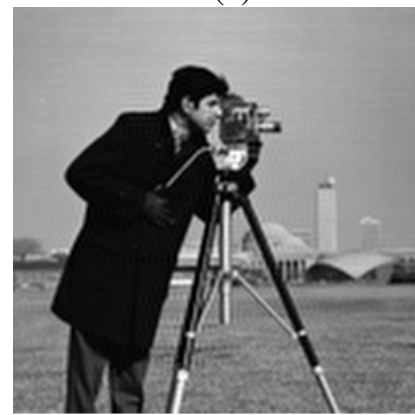

(c)

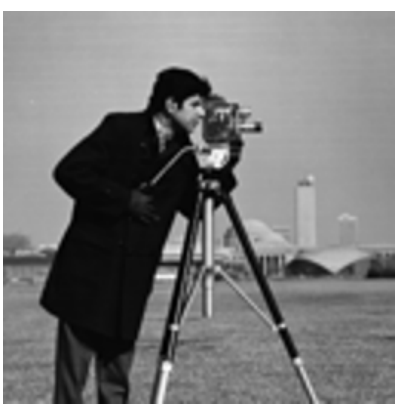

(b)

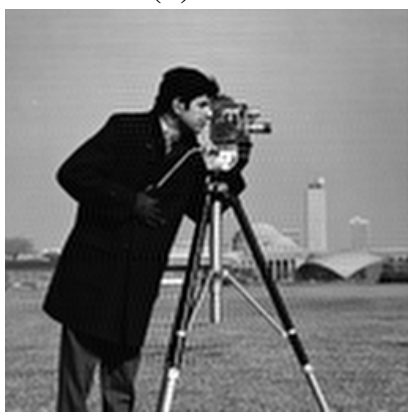

(d)

Fig.4: Subjective Evaluation of Cameraman using different Interpolation Techniques: (a) source image (b) Bicubic method (c) DCT method (d) Proposed Method.

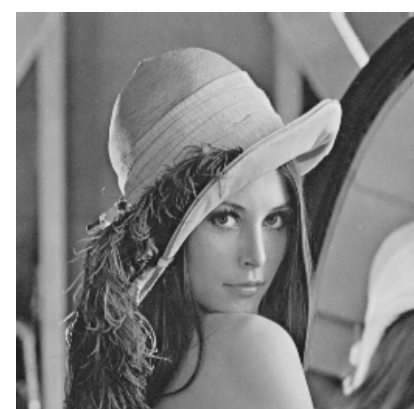

(a)

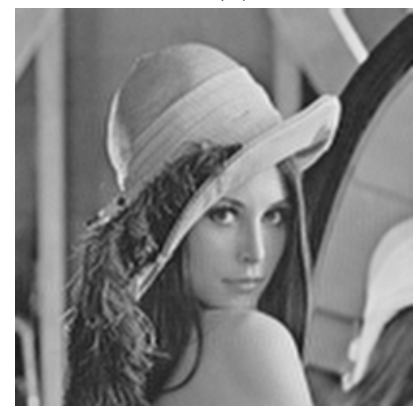

(c)

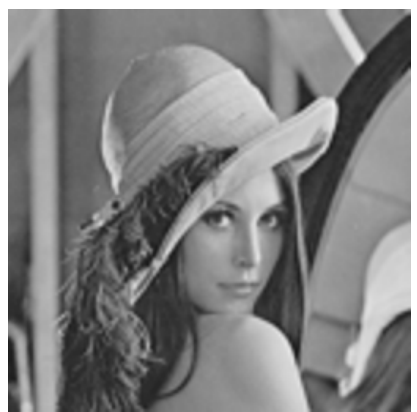

(b)

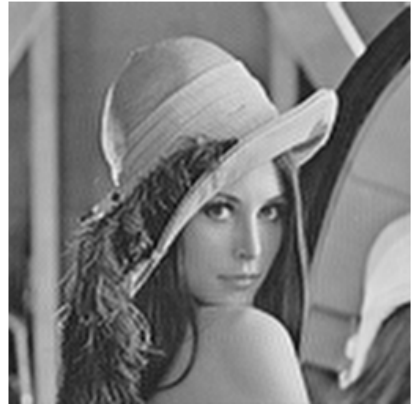

(d)

Fig.5: Subjective Evaluation of Lena using different Interpolation Techniques: (a) Source image (b) Bicubic method (c) DCT method (d) Proposed Method. 
International Journal on Information Theory (IJIT), Vol.4, No.1, January 2015

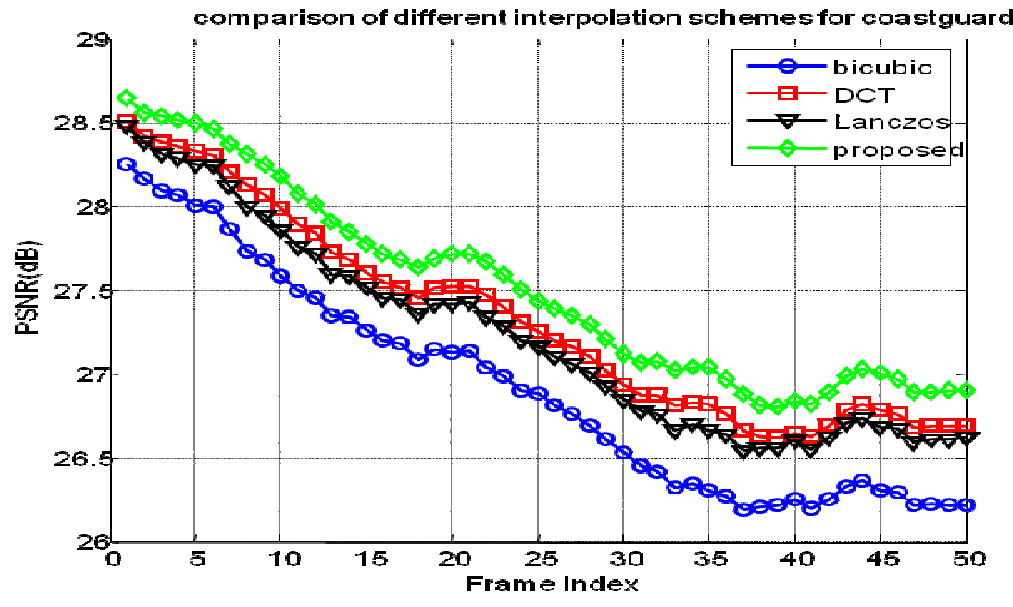

(a)

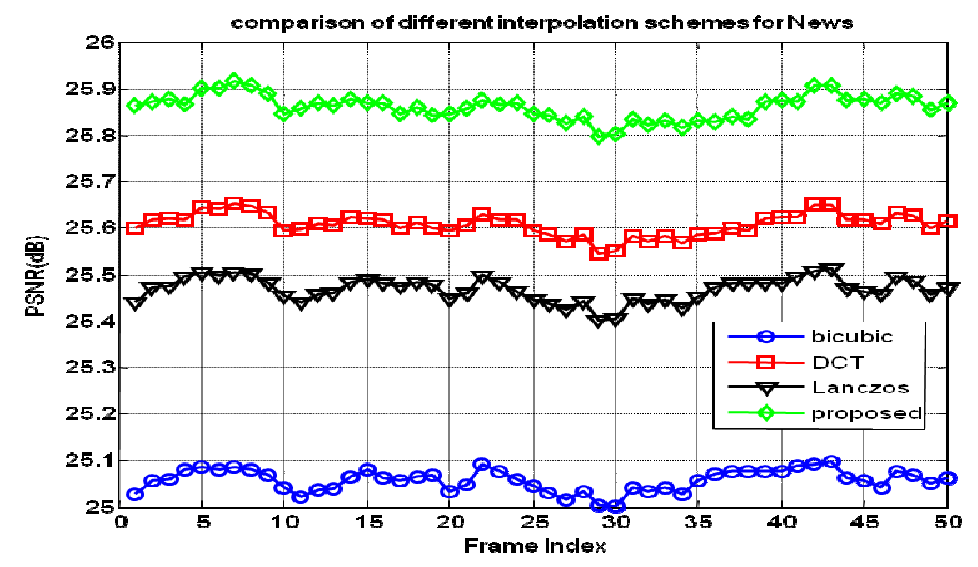

(b)

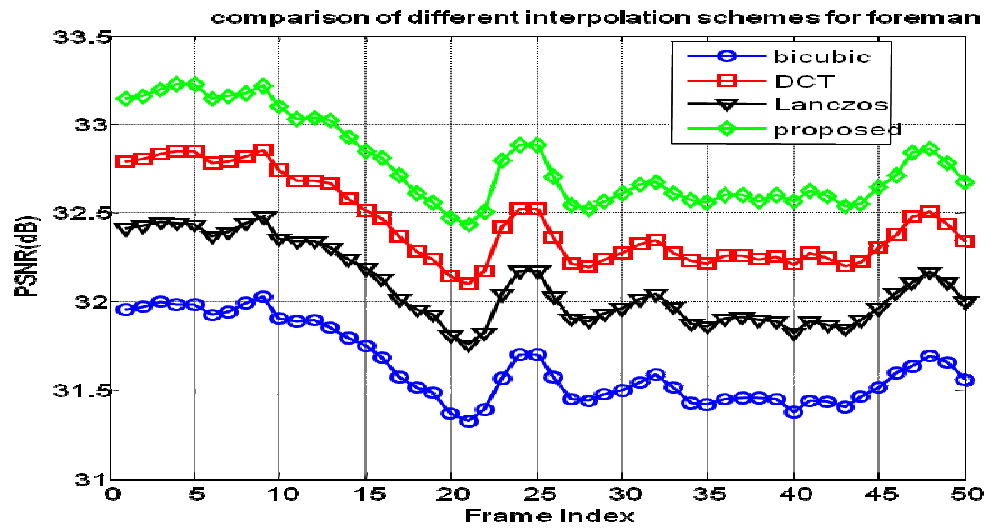

(c)

Fig.6. PSNR (dB) comparison of different sequences using different interpolation techniques at 4:1 compression ratio of :(a) coastguard (b) News (c) foreman. 
International Journal on Information Theory (IJIT), Vol.4, No.1, January 2015

Figure 6 represents the PSNR (dB) Vs frame index plot of different video sequences using various interpolation techniques at 4:1 compression ratio. Experimental results show, the proposed method yields better objective quality for different types of sequences having dissimilar characteristics.

\section{Conclusions}

In this paper the proposed algorithm exploits the advantages of both spatial domain and frequency domain processing for improved up-sampling performance in terms of PSNR $(\mathrm{dB})$. The proposed algorithm provides both subjective and objective improvement in comparison to other interpolation techniques irrespective of change in the resolution and compression ratio. In the reconstructed image the blurring is reduced considerably due to high frequency restoration through fusion based scheme. Furthermore, the proposed technique can also be adopted for local based and fuzzy based interpolation technique.

\section{ACKNOWLEDGEMENTS}

The authors would like to thank everyone, just everyone!

\section{REFERENCES}

[1] Lu Jing, Xiong Si, Wu Shihong, "An improved bilinear interpolation algorithm of converting standard defination images to high defination images," WASE International Conference on Information Engineering, pp. 441-444, 2009.

[2] R.G. Keys, "Cubic convolution interpolation for digital image processing," IEEE Trans. Acoust., speech, signal Processing, vol. ASSP- 29, no.6, pp. 1153-1160, Dec.1981.

[3] S.E. Reichenbach and F. Geng, "Two-dimensional cubic convolution," IEEE Trans. Image Processing, vol. 12, no.8, pp. 857-865, Aug. 2003.

[4] Zhou Dengwen, “An edge directed bicubic interpolation algorithm," CISP, pp. 1186-1189, 2010.

[5] G.Ramesh Babu and K.Veera Swamy, "Image Fusion using various Transforms," IPASJ International Journal of Computer Science (IIJCS), vol. 2, no.1, pp. 51-58, Jan. 2014.

[6] M.P.Parsai Deepak Kumar Sahu, "Different Image Fusion Techniques -A Critical Review," International Journal of Modern Engineering Research (IJMER), vol. 2, no. 5, pp. 4298-4301, Sep.Oct. 2012.

[7] Arpinder Singh Shaveta Mahajan, "A Comparative Analysis of Different Image Fusion Techniques," IPASJ International Journal of Computer Science (IIJCS), vol. 2, no.1, pp. 8-15, Jan. 2014.

[8] J. Mukherjee and S. K. Mitra, "Image resizing in the compressed domain using subband DCT," IEEE Trans. Circuits, Syst., Video Technol., vol. 12, pp. 620-627, July 2002.

[9] R. Dugad and N. Ahuja, "A fast scheme for image size change in the compressed domain," IEEE Trans. Circuits Syst. Video Technol., vol. 11, no.4, pp. 461-474, Apr. 2001.

[10] Zhenyu Wu, Hongyang Yu, and Chang Wen Chen, "A new hybrid DCT Wiener based interpolation scheme for video intraframe up-sampling," IEEE signal processing letters, vol. 17, no.10, pp. 827830, Oct. 2010. 